\title{
Automated Bumper for Damage reduction with Emergency Braking Mechanism
}

\author{
Aditya Gandhi ${ }^{*}$, Amey Deshmukh Rahul Aniyankunju and Prathamesh Kalasakar† \\ †Mechanical engineering, MITCOE Pune, Savitribai Phule Pune University, Pune, Maharashtra, India \\ Accepted 02 March 2016, Available online 15 March 2016, Special Issue-4 (March 2016)
}

\begin{abstract}
This paper is a hypothesis based on a very important requirement of safety in today's vehicles for both the vehicle and humans inside the vehicle around the globe. When implemented, It will be an added safety measure in the current facilities such as ABS, Air Bags, etc. The concept is to improve the damage reduction capacity by adding an extendable and retractable bumper with an emergency braking mechanism. This emergency brake can be used as a successive arrangement for the existing hand brake system. The assumption made in the concept is that the body of impact would be a perfectly rigid and stationary body. The bumper is supposed to absorb as much impact as possible with the use of various energy absorption materials. This paper features the concept and conceptual working schematic of the proposed vehicle. Simulation of this method can be done in order to visualize the results possible according to the required speed and mass of the vehicle under consideration.
\end{abstract}

Keywords: Vehicle safety etc.

\section{Introduction}

Even after all the added safety measures today the damage or fatality of the damage reduction is reduced by $60 \% .60 \%$ is not $100 \%$. Hence we are proposing a system to reduce the damage caused by an accident even further. The Automated Bumper and Emergency Braking System consist of pneumatic cylinders attacked to both the extendable bumper and the brake. These measures are required to stop the vehicle and reduce further damage possible if a collision occurs.

The bumper mechanism has been already tested by Mr. J.T. Wang, general Motors, USA in 2007. It was patented by Due to Doan in USA. He modified Aztek car with an extendable bumper provided with a tube structure for energy absorption purpose. We are proposing a model to couple this mechanism with an emergency braking mechanism to prevent the collision in the first place.

\subsection{Proposed model schematic}

This schematic shows the proposed arrangement of the entire mechanism. The E/R Bumper (Extendable and Retractable bumper) is fit to the car at the place of the existing bumper replacing it. A limit switch is added to actuate both the bumper and the emergency disk brake.

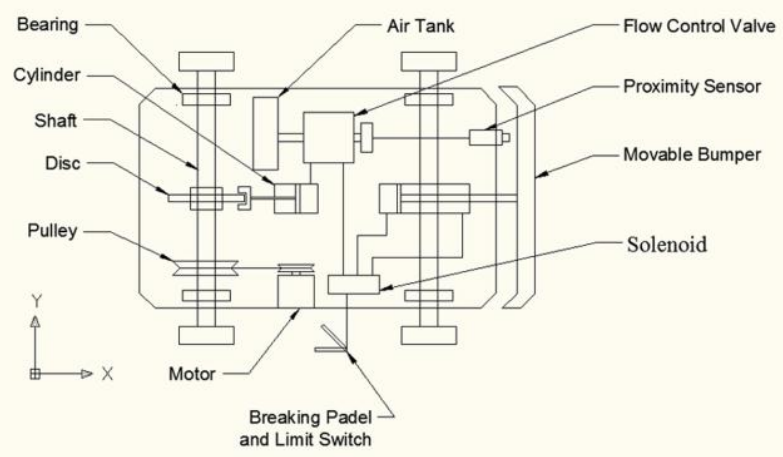

Fig.1 Schematic of the model

\section{Braking Mechanism}

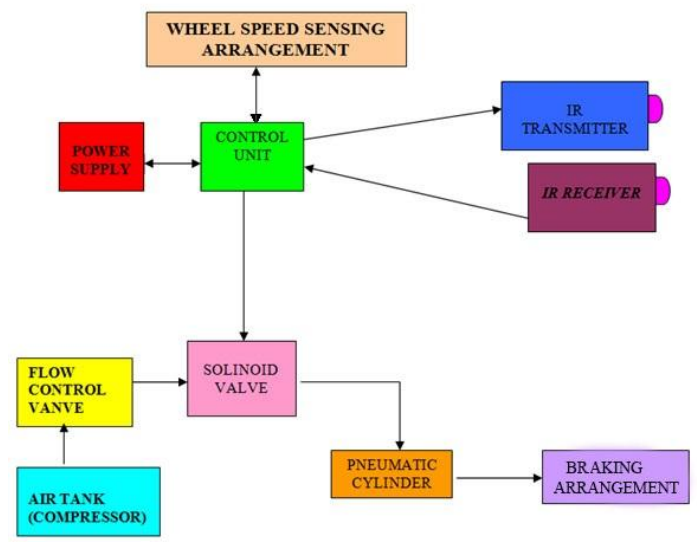


Our human tendency is to press the brake pedal as heavily as possible in case some obstacle comes in front of the car. Using this basic instinct we can add a limit switch at the back of the brake pedal.

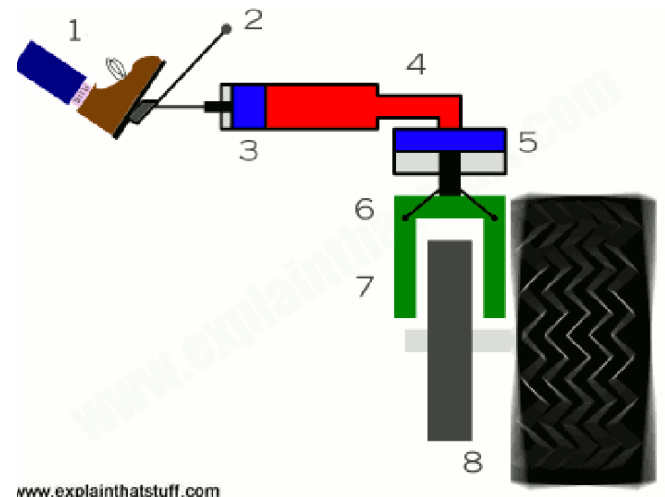

Fig 2.2. Pedal Braking Mechanism

Fig. 2.2 shows the normal braking mechanism used in any car. It is pedal brake system. When the pedal(2) is pressed by the driver(1), it compresses the fluid in the master cylinder(3-4-5) which then actuates the brake pads(6-7) to the braking $\operatorname{disk}(8)$.

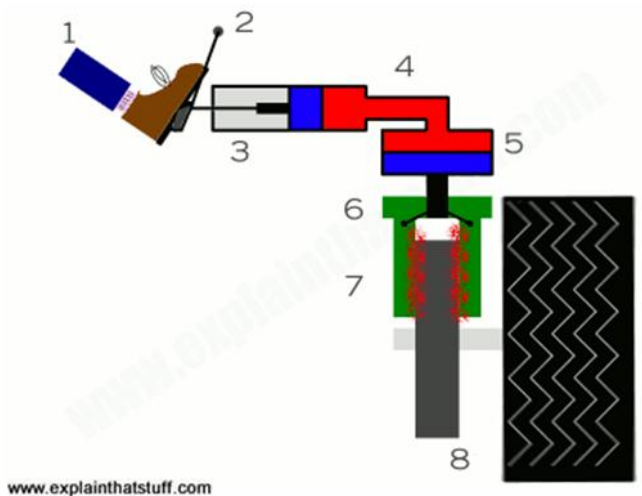

Fig 2.3. Brake in actuated condition

As mentioned before a human driver tends to push that pedal beyond the normal deflection angle of the brake pedal. Therefore we put a limit switch behind the brake pedal as shown in Fig 2.4.

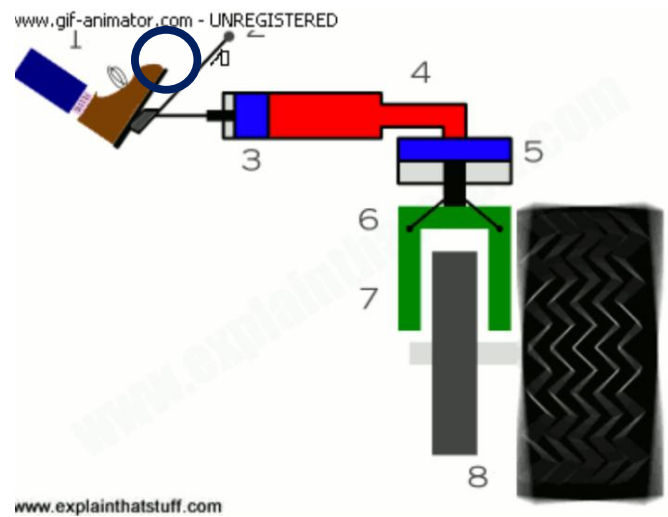

Fig 2.4 Limit Switch (Encircled) placed behind the brake pedal
As mentioned before when the brake pedal is pressed beyond the usual deflection angle, the limit switch is actuated which sends a signal to the control unit which centrally operates the emergency brake and the bumper mechanism.

The actuated limit switch can be shown as given in the Fig. 2.5. The figure also contains a representative arrow to show that the signal is sent to the control unit as soon as the limit switch is pressed.

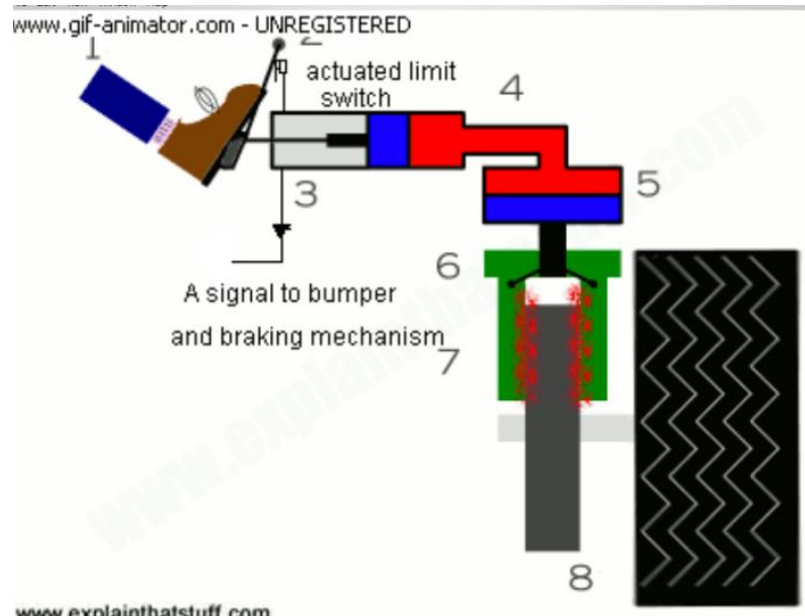

Fig 2.5 Actuated limit switch

\section{Working of the control unit}

A control unit is used in order to decide if the bumper should be actuated or not. A microprocessor can be programmed for this purpose. There are three inputs to the control unit (1) Speed Sensing unit, (2)Proximity sensor(to measure the distance between the moving vehicle and the stationary obstacle) and (3) signal from the limit switch.

This control unit will be activated as soon as the speed of the vehicle goes above $40 \mathrm{kmph}$. The speed is selected on the basis of a graph of Vehicle Speed vs Fatality of the Damage(shown in the graph 3.1).

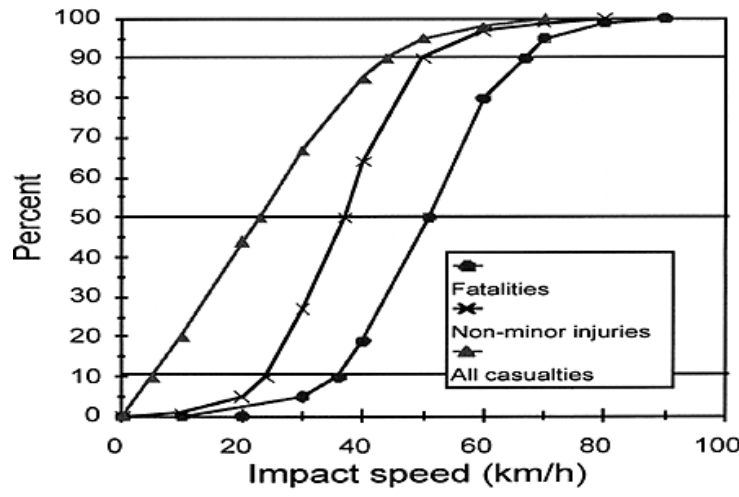

Graph 3.1 Impact speed Vs Fatality

It can be clearly seen from the graph that the fatality of damage from an accident goes on increasing as the speed of the vehicle goes on increasing. 
As soon as the control unit gents a signal from the limit switch it will couple all the inputs together i.e. distance between the vehicle and the obstacle and the speed of the vehicle and decide how much faster the bumper is required to act or if the emergency brake can stop the vehicle before impact .

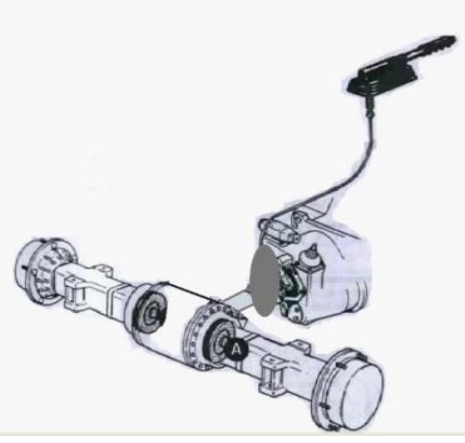

Fig 3.1Hand Brake working- normal state

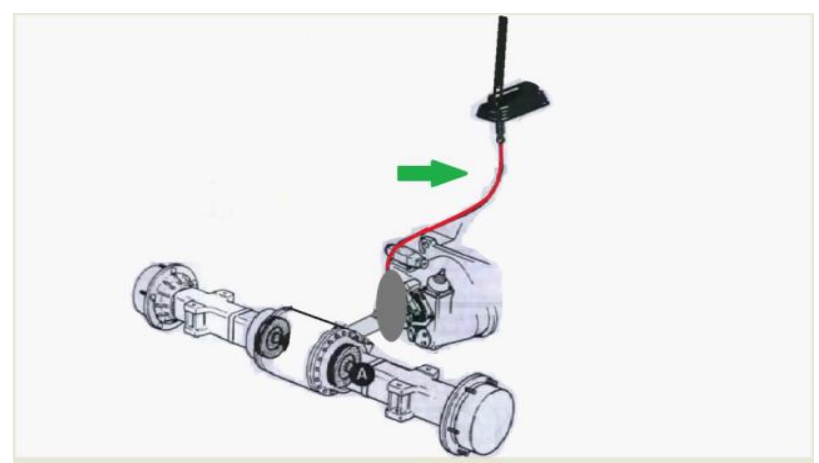

Fig 3.2Hand Brake working- energized state

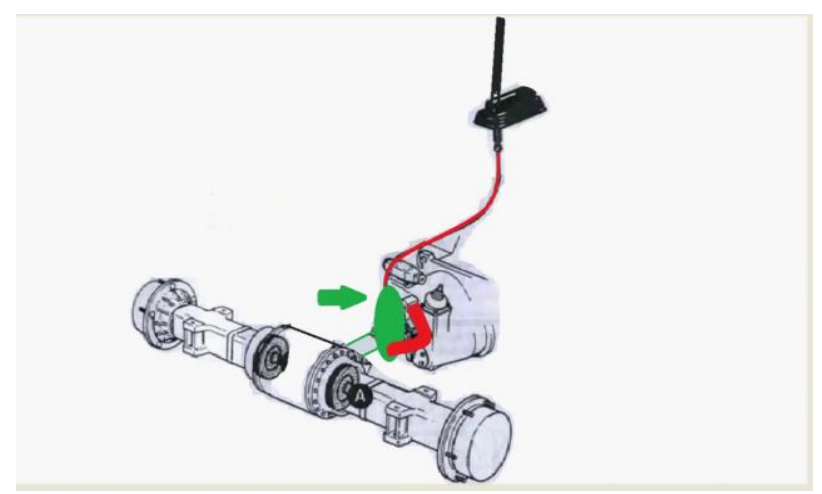

Fig 3.3Hand Brake working- wheel disk stopped

A microprocessor can be programmed using any appropriate language to perform as a control unit. Microprocessor are useful for this purpose as they can be programmed to perform the calculations required such as braking distance, time for activation, speed for actuation of the pneumatic pistons, how much air will be required to pass through the control unit etc.

Once these values are obtained it can compare the results and decide whether to actuate the bumper or not even though the limit switch is already actuated.

\section{Bumper Mechanism and its working}

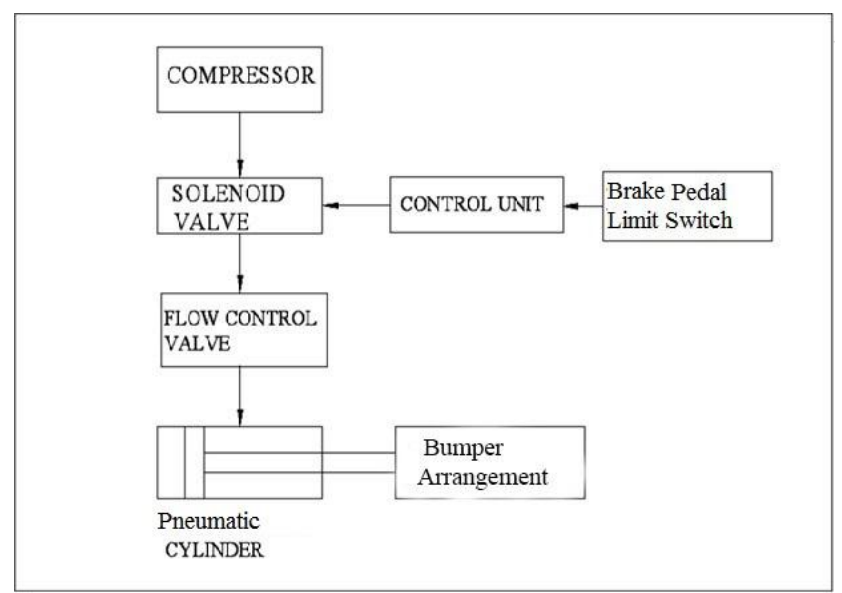

Fig. 4.1 Block Diagram for Bumper Mechanism part

The control unit has two decisions to make whether to actuate the bumper or not to actuate the bumper. This decision is made on the basis of the braking distance once the emergency brake is applied at a certain speed. Once the compared values indicate that the braking distance is more than the gap between the moving vehicle and the obstacle it decides to actuate the bumper to absorb the possible damage from the collision. The two conditions of the bumper are shown in the fig. 4.2 and fig 4.3 respectively. Fig/. 4.2 shows the condition when the bumper is not actuated where figure the fig. 4.3 shows the actuated bumper.

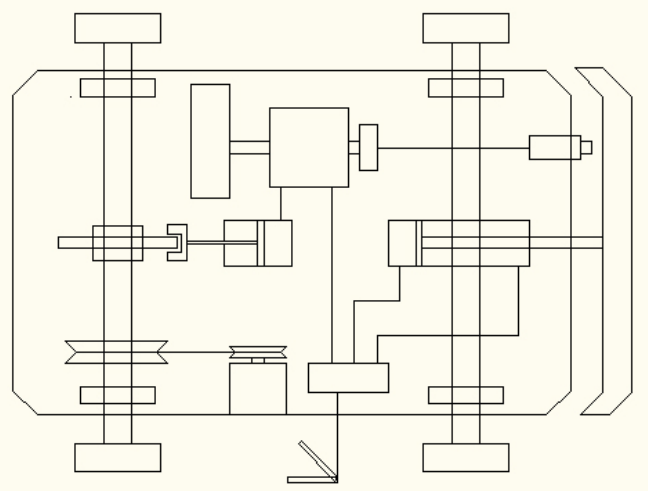

Fig 4.2 Bumper in non-actuated state

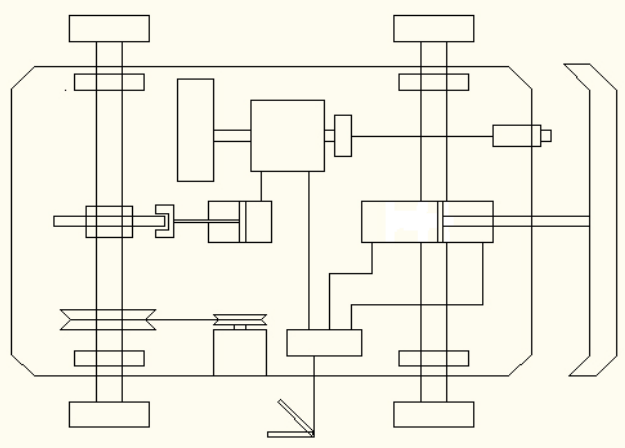

Fig. 4.3 Bumper in actuated state 
The bumper is lined with absorptive material to absorb the energy of the impact. Which in turn reduces the damage to the rest of the vehicle and the driver and/or passengers in the vehicle.

Once the control unit decides to actuate the bumper it sends a signal to the flow control valve which gives the required quantity if the air to the cylinder at which then actuates the bumper.

In both the cases for the brake and the bumper double acting cylinders are used so as it retract them with a reverse flow of the air to the cylinder piston.
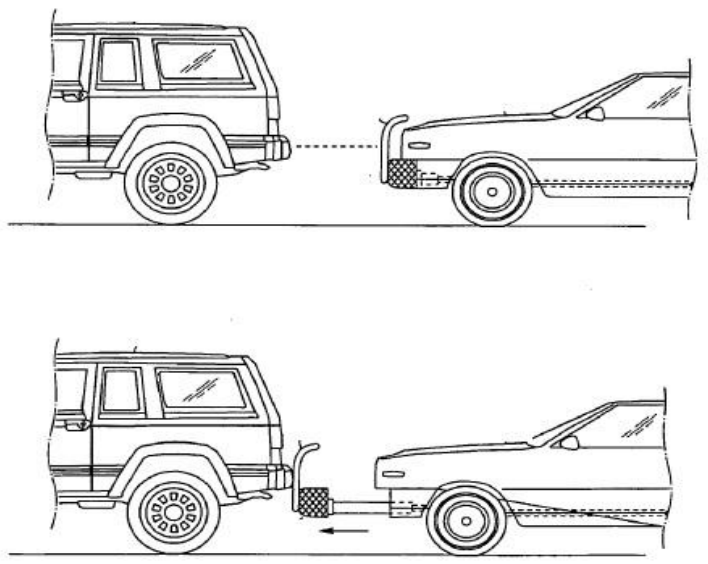

Fig. 4.4 Proposed movement of the bumper

Fig. 4.4 shows the proposed movement of the bumper before and after the collision once the control unit has decided to actuate the bumper.

\section{Conclusions}

These conclusions are based on hypothesis given above. The conclusions include both the advantages as well as limitations of this system.

1. Increasing the distance between the bumper and the vehicle the gradient of the vibration or jerk passing the backward direction can be reduced.
2. This gives additional safety to the vehicle and the driver and/or passengers.

3.The emergency braking is used as a successive arrangement to the available hand brakes.

4. The intelligent control unit is the key to the entire operation.

5. Use of an intelligent control unit i.e. the microprocessor can reduce the unnecessary wastage of energy through unwanted activation of the bumper or braking mechanism

6. Use of the control unit also features this mechanism as a reliable system

7. There is a huge amount of work needed to be done in order to design a self-sustaining system.

8. The system will increase the weight of the system , hence there is a need to balance the weight of the vehicle so as its C.G.(Center of Gravity) does not change.

9. Implementation of the system may require changing of the original design parameters of any given vehicle which would be a problem as this would increase the cost of design for the car manufacturers.

10.This will result in added cost of manufacturing which in the end will be affect the overall cost of the vehicle for a customer to buy.

11.The mechanism needs to be designed differently for each different vehicle as the weight will be different and the existing safety measures would be different for different models of vehicles,

\section{References}

Fatalities in Frontal Crashes Despite Seat Belts and Air Bags [U.S. Department of Transportation : DOT HS 811 202]

An Extendable And Retractable Bumper

[by J.T. Wang ,General Motors Corporation, United States, Paper No. 05-0144]

Extendable And Retractable Bumper Protection Apparatus,(2007)United States Patent Application Publication ,Pub. No.: US 2007/0290515 A1 Doan www.explainthatstuff.com/brakes.html http://www.safespeed.org.uk/killspeed.html 